\title{
On-Demand Transformation of Carbon Dioxide into Polymers enabled by comb shaped metallic oligomer catalyst
}

\author{
Han Cao ${ }^{1,2}$, Ruoyu Zhang ${ }^{1,2}$, Zhenzhen Zhou ${ }^{1,2}$, Shunjie Liu ${ }^{1,2}$, Youhua Tao ${ }^{1,2}$, Fosong Wang ${ }^{1,2}$, and Xianhong \\ Wang $^{1,2^{*}}$ \\ ${ }^{l}$ Key Laboratory of Polymer Ecomaterials, Changchun Institute of Applied Chemistry, Chinese Academy of \\ Sciences, Changchun, 130022, China \\ ${ }^{2}$ University of Science and Technology of China, Hefei, 230026, China \\ e-mail: xhwang@ciac.ac.cn
}

\begin{abstract}
:
Quantitative transformation of $\mathrm{CO}_{2}$ can greatly elevate the sustainability impact of $\mathrm{CO}_{2}$ chemical utilization, but it is formidably challenging due to the sluggish kinetics requiring overwhelmingly excess usage of $\mathrm{CO}_{2}$. Here, we report an on demand $\mathrm{CO}_{2}$ transformation by a switch polymerization method, that is, all reactants including $\mathrm{CO}_{2}$ are fully converted without any by-product, generating tailor-made poly(ether carbonate) polyols $\left(\mathrm{CO}_{2}\right.$-polyols) whose composition and chain length exactly correspond to the feed of $\mathrm{CO}_{2}$, epoxide and diacid. This is the first time for $\mathrm{CO}_{2}$ as a countable monomer which is in most cases obscurely considered as "pressure condition". Studies on the kinetics rate law and the activation parameters of key intermediates disclose that it is the multisite cooperativity from metallic oligomer catalyst that facilitates quantitative insertion of $\mathrm{CO}_{2}$ into polymer backbone without adverse backbiting throughout the polymerization. Hence, this work not only introduces the conception of quantitative $\mathrm{CO}_{2}$ transformation, but engineers exquisite $\mathrm{CO}_{2}$-based polymer which is rarely achieved.
\end{abstract}

Keywords: quantitative, $\mathrm{CO}_{2}$ transformation, $\mathrm{CO}_{2}$-polyol, oligomer catalyst, precise catalysis

\section{Introduction}

Chemical conversion of $\mathrm{CO}_{2}$ offers a promising avenue to valorize the waste "greenhouse" gas and deviate the reliance of chemical synthesis on fossil fuel feedstocks, thus creating a more sustainable carbon economy. ${ }^{1,2}$ However, the common high pressure reaction condition results in low $\mathrm{CO}_{2}$ conversion, leading to either the discharge of troublesome $\mathrm{CO}_{2}$ to the atmosphere, or the cumbersome energy input $\mathrm{CO}_{2}$ recycle. To date, only few reactions can theoretically consume $\mathrm{CO}_{2}$ in quantitative yield, such as catalytically irrelevant mineral carbonation ${ }^{3}$ and amines to absorb $\mathrm{CO}_{2}$ and generate carbamate. ${ }^{4}$ But quantitative $\mathrm{CO}_{2}$ transformation is scarcely reported in sophisticated $\mathrm{CO}_{2}$ catalysis such as various $\mathrm{CO}_{2}$ reduction reactions to afford fuels and bulk chemicals, ${ }^{5,6}$ and non-redox coupling reactions for cyclic carbonate and polymer production. ${ }^{7-9}$ The product yield or the conversion of economically valuable reactants is at the center of these research fields while $\mathrm{CO}_{2}$ conversion is neglected and hardly studied on a mole basis. In this context, we are wondering whether $\mathrm{CO}_{2}$ could be quantitatively consumed in these advanced $\mathrm{CO}_{2}$ chemical transformations. 


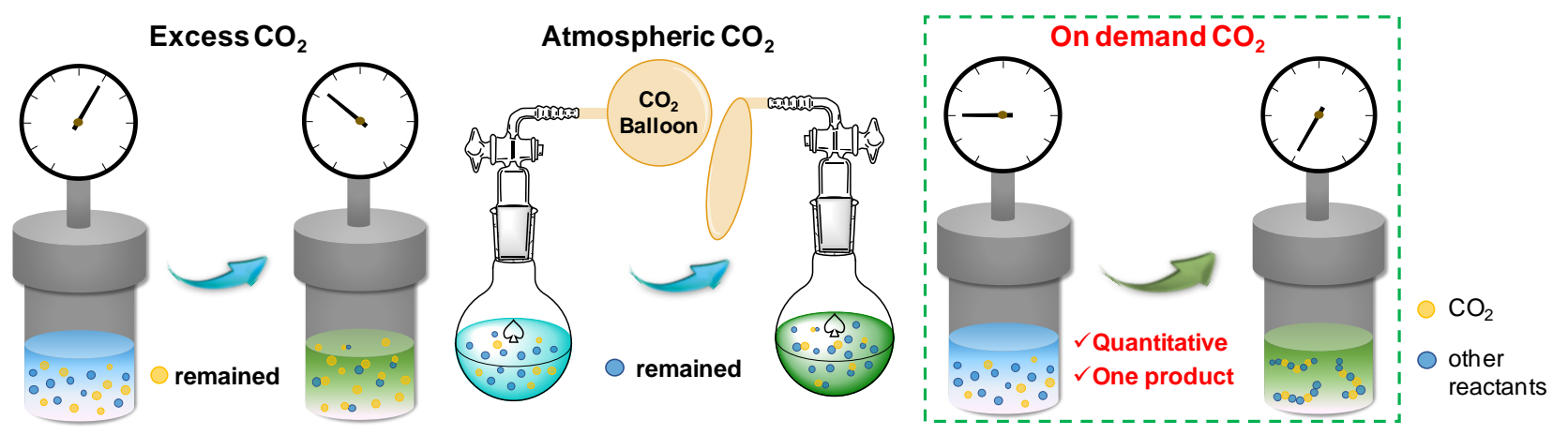

Figure 1. Conceptual blueprint of on demand $\mathrm{CO}_{2}$ transformation.

Technically, quantitative transformation of $\mathrm{CO}_{2}$ can be simply achieved by just feeding less. Taking the cycloaddition reaction as example, ambient pressure $\mathrm{CO}_{2}$ can be fully reacted with largely excess epoxide, which obviously puts the cart before horse as the great surplus of carbon-containing compounds. ${ }^{10}$ Meanwhile, the increased conversion in $\mathrm{CO}_{2}$ reaction can also diminish otherwise well-controlled selectivity and thus form undesired product distribution. ${ }^{11}$ Energy required in product separation leads to extra $\mathrm{CO}_{2}$ production. Therefore, simple full consumption of $\mathrm{CO}_{2}$ without strict criteria is not the objective here. Instead, what we desire to establish is a comprehensive scenario which lets all reactants serve their proper purpose. That is, an on demand $\mathrm{CO}_{2}$ transformation is proposed which is different from both the lavish high-pressure reaction, and also the pursuit of absolute ambient pressure reaction (Fig. 1). The term "on demand" here refers to: 1) complete conversion of every involved reactants and 2) only one product formed. Such scenario of "make the best use of everything" renders $\mathrm{CO}_{2}$ catalysis in line with the principles of green chemistry to a larger extent. ${ }^{12}$

In this work, we successfully fulfill the on demand $\mathrm{CO}_{2}$ transformation by virtue of the catalytic chain-transfer polymerization of $\mathrm{CO}_{2}$ and propylene oxide (PO). The reaction that generates cutting-edge $\mathrm{CO}_{2}$-derived product the poly(ether carbonate) polyol, ${ }^{13-15}$ provides a platform for our proposal from three perspectives: for reaction formula, $n$ $\mathrm{CO}_{2}$ molecules are fixed as carbonate linkage via ring-opening copolymerization (ROCOP) with equivalent POs, and ring-opening polymerization (ROP) of remained $\mathrm{m}$ POs form ether linkage; for mechanism, the insertion of $\mathrm{CO}_{2}$ is fast and zero-order dependent in rate law; ${ }^{16-18}$ for reaction system, the soft ether linkage and the nature of formed low-molarmass polymer allow the full conversion without the restriction from viscosity. Notwithstanding the theoretical viability, such "on demand" $\mathrm{CO}_{2}$ transformation via polyol route is challenging from catalyst. State-of-the-art $\mathrm{CO}_{2} /$ epoxide ROCOP catalysts, either stringently afford carbonate linkage which gives rise to solidified medium limiting full conversion, ${ }^{19-21}$ or only exhibit activity at harsh condition inducing high proportion of $\mathrm{CO}_{2}$ in the gas phase and sidereaction. ${ }^{22-24}$ To address this issue, we adopt a comb shaped metallic oligomer catalyst CAT 1 comprising multiple functionalized $\mathrm{Al}(\mathrm{III})$ porphyrin complexes at the side chain. Owing to the unique multimetallic synergistic catalysis, CAT 1 exhibits fast and comparable ROCOP and ROP rate with unprecedented selectivity, which quantitatively converts $\mathrm{PO}$ and $\mathrm{CO}_{2}$ into $\mathrm{CO}_{2}$-polyols as the sole and only target in a switch catalysis manner (Fig. 2). This proof-of-concept study offers a fresh perspective on precisely handling $\mathrm{CO}_{2}$ in reactions and also a more convincing blueprint of sophisticated $\mathrm{CO}_{2}$ catalysis as a potential toolbox for future carbon reduction. 


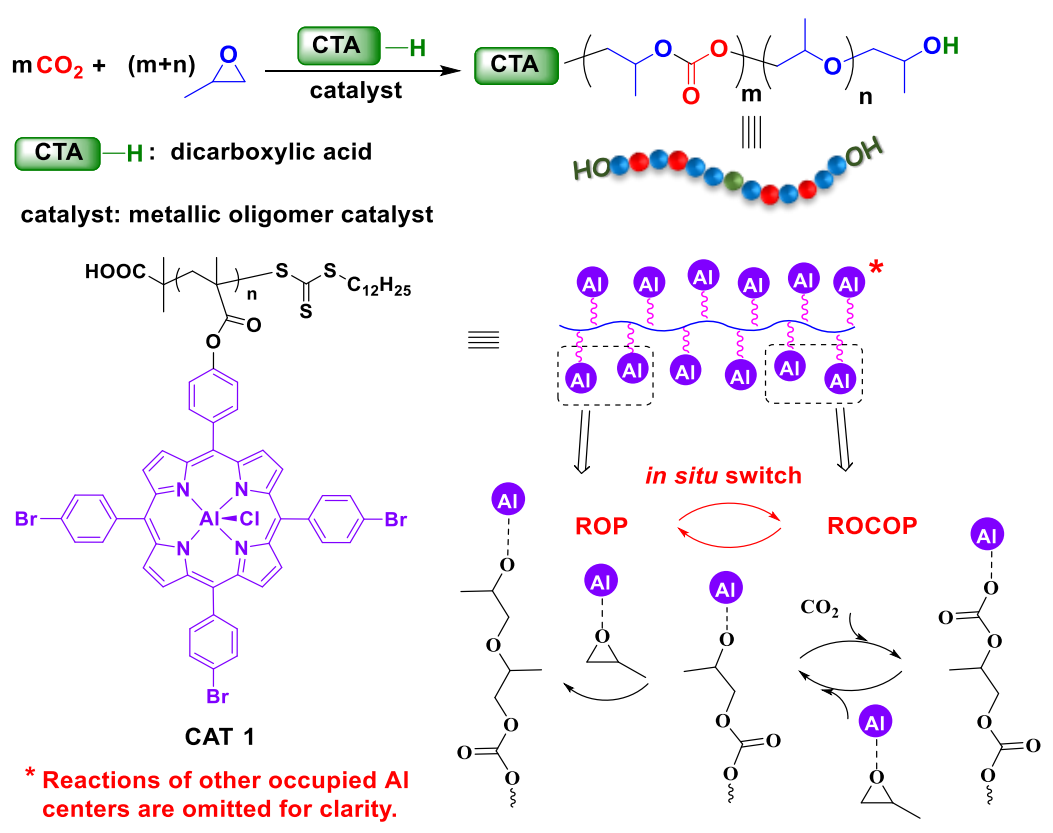

Figure 2. On demand $\mathrm{CO}_{2}$ transformation realized in the chain-transfer polymerization of epoxide and $\mathrm{CO}_{2}$ using a comb shaped metallic oligomer catalyst (CAT 1).

\section{Results and discussion}

\section{Tentative attempt}

The first objective of our approach is screening catalyst that enables to polymerize $\mathrm{CO}_{2}$ and excess PO to polyols in full conversion without any by-product, i.e. on demand transformation. The by-product refers to cyclic propylene carbonate (cPC) which is often generated in $\mathrm{PO} / \mathrm{CO}_{2} \mathrm{ROCOP}$ reaction. The first candidate is double metal cyanide (DMC) solid catalyst pertain to large-scale synthesis of poly(ether carbonate) polyols. ${ }^{22,23}$ However, literatures report that it often operates under high pressure $(>2 \mathrm{MPa})$ and temperature $\left(>80^{\circ} \mathrm{C}\right)$ unable to assure full conversion of $\mathrm{CO}_{2}$. Alternatives are discrete molecular ROCOP catalysts. Some of them are active at low and even ambient $\mathrm{CO}_{2}$ pressure, but whether quantitative conversion is achievable has not been distinctly demonstrated. ${ }^{25,26}$ In our initial hypothesis, although these catalysts in priority alternatively copolymerize $\mathrm{PO}$ with $\mathrm{CO}_{2}$, full monomer enchainment can be realized provided switch catalysis occurs from ROCOP to PO ROP in substoichiometric $\mathrm{CO}_{2}$ feed. Thus, we initially test this hypothesis using $\mathrm{Al}(\mathrm{III})$ porphyrin complex (mono 1) by monitoring its whole kinetics profiles with $1.0 \mathrm{MPa} \mathrm{CO}_{2}$ feed (substoichiometric) and comparing to those with excess $\mathrm{CO}_{2}(3.5 \mathrm{MPa})$.

According to aliquot ${ }^{1} \mathrm{H}$ NMR analysis (Fig. 3a), the stoichiometry of $\mathrm{CO}_{2}$ did not influence the rate since the conversion of PO was basically synchronous in the two reactions, however in sharp contrast, the reaction at $3.5 \mathrm{MPa}$ only generated $\mathrm{Ca} .3 \mathrm{wt} \%$ of $\mathrm{cPC}$ in invariant selectivity with time while the reaction at $1.0 \mathrm{MPa}$ displayed two distinct stages. When $\mathrm{CO}_{2}$ is sufficient, the selectivity towards $\mathrm{cPC} /$ polyol showed similar with that at high pressure. However, huge amount of cPC (24 wt\%) was somehow generated in the end. It was calculated that the ratio of fixed $\mathrm{CO}_{2}$ in polyols and $\mathrm{cPC}$ was $1 / 1.38$ implying the big sacrifice towards selectivity only for the purpose of exhausting $\mathrm{CO}_{2}$. A separate study using in situ infrared spectroscopy disclosed that at high $\mathrm{CO}_{2}$ conversion the hypothesized switch catalysis did not occur (Fig. 3b-c). The major reaction was polyols depolymerizing to cPC via back-biting while the switch to ROP was the minority. As shown in Table S1, single-site Al(III) porphyrin catalysts were failed to our goal regardless of the 
variation of substitution in the porphyrin ligand. Moreover, similar result was also observed using Co(III) salen catalyst (Table S1, Entry 5). ${ }^{20}$ Thus, initial attempts using common mononuclear catalysts were unsuccessful.
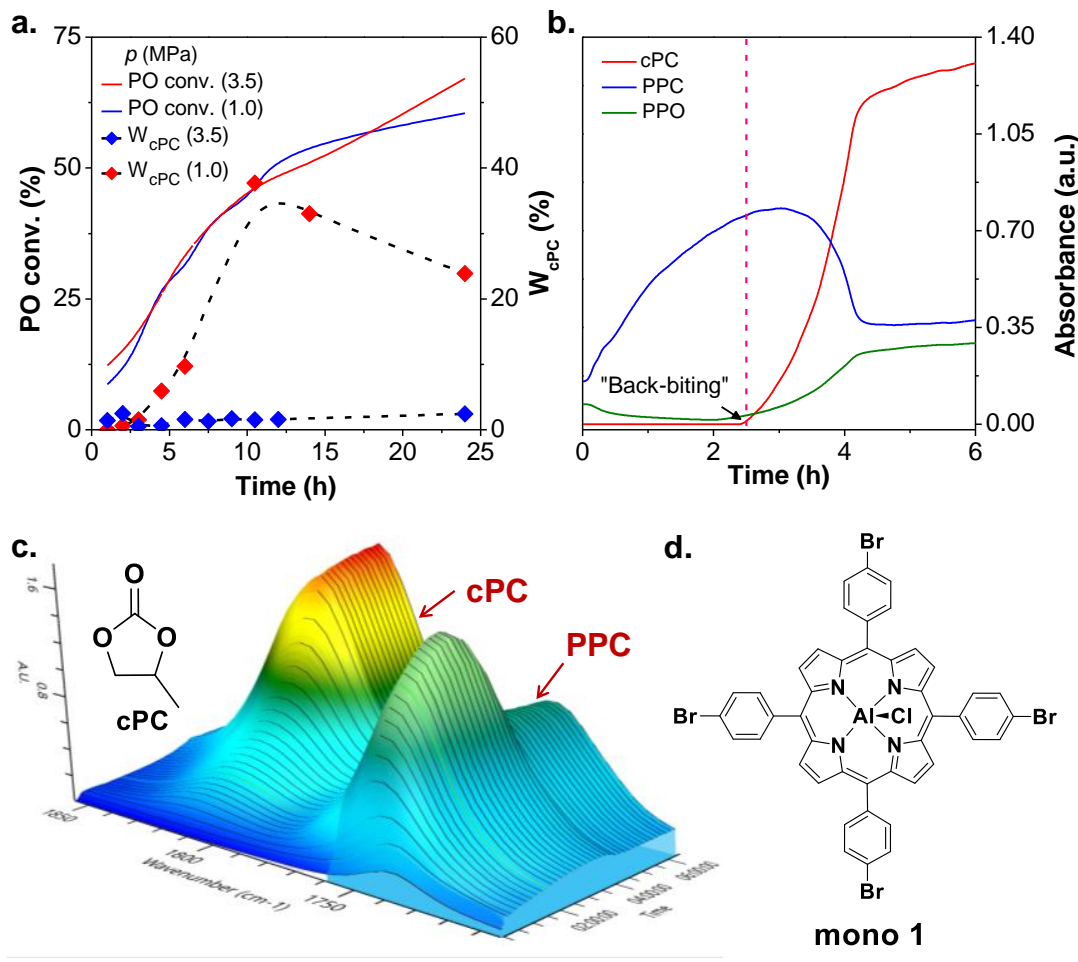

Figure 3. Monitoring quantitative $\mathrm{CO}_{2}$ polymerization catalyzed by mononuclear complex (mono 1). a. PO conversion and $\mathrm{cPC}$ content in polyols, monitored by ex situ aliquot ${ }^{1} \mathrm{H}$ NMR analysis. Reaction conditions: $\mathrm{PO} /[\mathrm{mono}$ 1]/PPNCl=2500/1/1\& $\mathrm{PO} / \mathrm{SA}=30$ (molar ratio), $70{ }^{\circ} \mathrm{C}, 1.0 \mathrm{MPa} / 3.5 \mathrm{MPa} \mathrm{CO}_{2}$ pressure. The details are listed in Fig. S2S5. b and c. Reaction profiles monitored by in situ IR and the resulting three-dimensional stack plot of the IR spectra. Profiles of the absorbance at $C a .1800 \mathrm{~cm}^{-1}$ corresponds to $v(\mathrm{C}=\mathrm{O})$ from cPC. Profiles of the absorbance at $C a .1745 \mathrm{~cm}^{-}$ ${ }^{1}$ corresponds to $v(\mathrm{C}=\mathrm{O})$ from linear carbonate $(\mathrm{PPC})$ and $\mathrm{C}=\mathrm{O}$ from ester (formatted from $\mathrm{SA}$ ) in polyols. Profiles of the absorbance at $C a .1104 \mathrm{~cm}^{-1}$ corresponds to $v(\mathrm{C}-\mathrm{O})$ from ether (PPO) in polyols. Reaction condition: $\mathrm{PO} /[\mathrm{SA}] /[\mathrm{mono}$ 1] $/ \mathrm{PPNCl}=2500 / 50 / 1 / 1 \& \mathrm{PO} / \mathrm{SA}=30$ (molar ratio), $70{ }^{\circ} \mathrm{C}, 1.5 \mathrm{MPa}$. d. Chemical structure of mono 1.

\section{Metallic oligomer catalyst CAT 1.}

With the above results in mind, we consider that the catalyst here should enable 1) fast ROCOP and ROP processes and in situ switch between them; 2) excellent protic CTA compatibility for chain-transfer; 3) permanent prevention of backbiting side-reaction. Recently, our group reported a series of metallic oligomer catalysts composed of Ca. 4-7 Al(III) porphyrin complexes at the side chain, which greatly suppressed cPC formation in the copolymerization of $\mathrm{PO}$ and $\mathrm{CO}_{2}$ and yielded high-molar-mass poly(ether carbonate). ${ }^{27}$ Relatively, in the presence of 50-100 equivalent water and carboxylic acids, the published CAT $\mathbf{0}$ achieved excellent selectivity, generating $\mathrm{CO}_{2}$-polyols with controllable molar mass and narrow distribution (Table S2). However, as a candidate for our task, its activity no matter for turnover frequency (TOF) or productivity needs further improvement. Like most organometallic catalysts, the molecularly welldefined active sites and tailorable structure permit an incisive mechanism-based performance optimization. In this work, several structural modifications considering the identity and number of organometallic moieties, the main chain and linker constructing the oligomer, were carried out as follows: 1) The bromide was introduced in the meso-position of the 
porphyrin ring to modulate the Lewis acidity of the metal center. ${ }^{28}$ 2) The linker between the porphyrin and the main chain was shortened to facilitate the reaction between metal-monomer and metal-chain end. 3) The number of porphyrins per chain was increased to $C a .12$ to further enlarge the multisite cooperativity (Fig. S12). Finally, CAT 1 was obtained in a four-step synthesis (Fig. S13). Its catalytic performance was distinctly outstanding. At $80{ }^{\circ} \mathrm{C}$, TOF of 9,600 h-1 was observed in CAT 1 catalyzed chain-transfer polymerization of $\mathrm{PO}$ and $\mathrm{CO}_{2}$ in the presence of sebacic acid (SA), which was $C a .40$ times higher than mono 1 under the same condition (Entry 1 vs 7, Table S4). Note that for better comparison with single-site catalysts, TOF was calculated based on the substrate conversion per Al center. By raising temperature to $120^{\circ} \mathrm{C}$, TOF of $17,600 \mathrm{~h}^{-1}$ was obtained within $1 \mathrm{~h}$, exhibiting very competitive activity compared with the state-of-theart organometallic $\mathrm{CO}_{2}$ polymerization catalysts, such as $\mathrm{Al}(\mathrm{III})$ porphyrin ${ }^{29}$ and $\mathrm{Co}(\mathrm{III})$ salen $^{11}$ in a bifunctional fashion, and $\mathrm{Mg}(\mathrm{II}) / \mathrm{Co}(\mathrm{II})$ heterodinuclear complex ${ }^{25}$. For comparison with solid catalyst, the value of productivity is also introduced, based on the production of polyols per gram of catalyst. By controlling the temperature, a productivity of 12.5 ( $\mathrm{kg}$ polyol g${ }^{-1}$ cat. $^{-1}$ ) was achieved for $24 \mathrm{~h}$ (Entry 9, Table S4). As for selectivity, CAT 1 cut down the formation of $\mathrm{cPC} \%$ below $1 \%$ at $80{ }^{\circ} \mathrm{C}$ and reduced temperature lead to even lower cPC content. Thus, compared with DMC, CAT 1 demonstrates similar or improved productivity and distinctly advanced selectivity. ${ }^{30,31}$ Hence, the cutting-edge performance of CAT 1 will support our further exploration on our unfinished work.

\section{CAT 1 mediated quantitative $\mathrm{CO}_{2}$ polymerization.}

The results of CAT 1 mediated on demand $\mathrm{CO}_{2}$ transformation via polyol route were shown in Table 1. For selectivity, the formation of $\mathrm{cPC}$ was all below $0.5 \mathrm{wt} \%$ indicating that the hitherto ineluctable backbiting reaction did not occur. As for conversion, final crude mixture of each run was viscous but flowable liquid rather than waxy solid as a consequence of low molar mass and carbonate ratio, facilitating the complete monomer conversion in such bulk condition. Particularly, for entry 1-12, the pressure reading of autoclave fell to minimum in the end and no $\mathrm{CO}_{2}$ bubbles left when stirring the crude mixture. By quantitative calculation, the conversion of $\mathrm{CO}_{2}$ all exceeded $94 \%$ (the initial $\mathrm{CO}_{2}$ usage was recorded by mass for careful calculation). Hence, the nearly complete consumption of both monomers and the afforded polyols as the sole product demonstrated that our proposed on demand transformation was successful. Meanwhile, the above experiments with fast polymerization rate were all conducted at ppm level catalyst concentration, where the molar ratio of $[\mathrm{Al}] /[\mathrm{PO}]$ was $1 / 50,000 /$ that corresponds to [CAT 1]/[PO] of $C a$. 1/600,000. Such low catalyst loading minimizes the organometallic residue and further elevate the sustainability impact of this work from the perspective of green synthesis.

An equally important finding is that on demand transformation of $\mathrm{CO}_{2}$ in turn favored the precise tuning of polyol composition. As shown in Fig. 4a, there was a good linear fit between the initial ratio of monomers and the composition of the polymer in the quantitative zone, where the carbonate ratio in polyol spaciously ranged from $6.9 \%-36.8 \%$, one-toone corresponding to initial $\mathrm{CO}_{2} / \mathrm{PO}$ of $0.07-0.39$. The threshold of quantitative zone is related to the intrinsic ROCOP/ROP selectivity of CAT 1 which is discussed later. Remarkably, this is the first time for using $\mathrm{CO}_{2}$ as a countable monomer to produce polymers with predictable carbonate ratio in the field of $\mathrm{CO}_{2}$ polymer chemistry. As previously reported by us, the composition of $\mathrm{CO}_{2}$-polyols was only roughly tuned by the reaction condition since heterogeneous catalysis did not allow precise control. ${ }^{23,31}$ In comparison with another previous literature, where similar range of carbonate linkage with Table 1 here was obtained at $90{ }^{\circ} \mathrm{C}$ and $15-90$ bar $\mathrm{CO}_{2}$ pressure, ${ }^{14} \mathrm{CAT} 1$ produced $\mathrm{CO}_{2}$-polyols with much lower energy input, which also coupled with the advance of purification-free towards cPC residue. Currently, $\mathrm{CO}_{2}$-polyols with less $\mathrm{CO}_{2}$ uptake are the drop-in solution for replacing the fully petroleum-derived polyether polyols in 
the manufacturing of polyurethanes, which is a rare example for $\mathrm{CO}_{2}$ catalysis already in commercial process. ${ }^{32}$ Due to the tunable composition of ether/carbonate, $\mathrm{CO}_{2}$-polyol retains the traits of polyether polyol such as low viscosity and favorable glass transition temperature $\left(T_{\mathrm{g}}\right)$ as well as endows the derived polyurethane more comprehensive material property. ${ }^{33,34}$ In this unique on demand transformation, $\mathrm{CO}_{2}$-consumed synthesis and $\mathrm{CO}_{2}$-derived products offer mutual benefit for each other, i.e., a $\mathrm{CO}_{2}$-polyol route can directly realize the quantitative conversion of $\mathrm{CO}_{2}$, and complete conversion provides a made-to-order synthetic protocol for exquisite $\mathrm{CO}_{2}$-derived polymer.

Table 1. Results of on demand $\mathrm{PO} / \mathrm{CO}_{2}$ polymerization catalyzed by CAT 1

\begin{tabular}{ccccccccccc}
\hline entry $^{\mathrm{a}}$ & $\begin{array}{c}\mathrm{CO}_{2} \\
\text { ini. }[\mathrm{g}]\end{array}$ & $\begin{array}{c}\mathrm{CO}_{2} / \\
\mathrm{PO}^{\mathrm{b}}\end{array}$ & $\begin{array}{c}\mathrm{Conv} \text {. of } \\
\mathrm{PO}[]^{\mathrm{c}}\end{array}$ & $\begin{array}{c}\mathrm{W}_{\mathrm{cPC}}^{\mathrm{d}} \\
{[\mathrm{wt} \%]}\end{array}$ & $\begin{array}{c}\mathrm{TOF} \\
{\left[\mathrm{h}^{-1}\right]^{\mathrm{e}}}\end{array}$ & $\begin{array}{c}\text { Carbona } \\
\text { te }[\%]^{\mathrm{f}}\end{array}$ & $\begin{array}{c}\mathrm{CO}_{2} \text { in } \\
\text { polyol }[\mathrm{g}]^{\mathrm{g}}\end{array}$ & $\begin{array}{c}\mathrm{CO}_{2} \text { fixed } \\
{[\%]^{\mathrm{g}}}\end{array}$ & $\begin{array}{c}M_{n}^{\mathrm{h}} \\
{[\mathrm{g} / \mathrm{mol}]}\end{array}$ & $\Xi^{\mathrm{h}}$ \\
\hline 1 & 1.98 & 0.07 & $>99$ & 0.2 & 2500 & 6.9 & 1.96 & $>99$ & 3200 & 1.11 \\
3 & 2.76 & 0.11 & $>99$ & 0.1 & 2500 & 10.6 & 2.66 & 96.3 & 3500 & 1.10 \\
4 & 4.05 & 0.16 & $>99$ & 0.4 & 2500 & 15.6 & 3.92 & 96.8 & 3500 & 1.11 \\
5 & 5.30 & 0.21 & 94.5 & 0.2 & 2360 & 22.0 & 5.23 & 98.7 & 3600 & 1.12 \\
6 & 6.33 & 0.25 & 98.6 & 0.2 & 1970 & 24.6 & 6.10 & 96.4 & 3600 & 1.11 \\
7 & 7.37 & 0.29 & $>99$ & 0.4 & 1670 & 28.9 & 7.27 & 98.6 & 3700 & 1.11 \\
$8^{\mathrm{i}}$ & 7.20 & 0.29 & $>99$ & 0.4 & 1670 & 27.5 & 6.92 & 96.1 & 3000 & 1.10 \\
$9^{\mathrm{j}}$ & 7.04 & 0.28 & $>99$ & 0.4 & 1670 & 27.1 & 6.82 & 96.9 & 2500 & 1.11 \\
$10^{\mathrm{k}}$ & 7.29 & 0.29 & $>99$ & 0.5 & 1670 & 28.4 & 7.14 & 97.9 & 1900 & 1.10 \\
11 & 8.89 & 0.35 & $>99$ & 0.3 & 1670 & 34.1 & 8.58 & 96.5 & 3800 & 1.12 \\
12 & 9.81 & 0.39 & 95.6 & 0.4 & 1590 & 36.8 & 9.26 & 94.4 & 3800 & 1.11 \\
13 & 11.72 & 0.47 & $>99$ & 0.2 & 1250 & 40.2 & 10.06 & 85.8 & 4000 & 1.12 \\
$14^{1}$ & 5.89 & 0.47 & 94.8 & 0.8 & 590 & 44.9 & 5.35 & 90.8 & 3900 & 1.11 \\
15 & 13.71 & 0.54 & $>99$ & 0.4 & 1250 & 43.4 & 10.85 & 79.1 & 4000 & 1.12 \\
\hline
\end{tabular}

a. Polymerization reactions were run with molar ratio of [PO]:[SA]:[Al]:[PPNCl] $=50000: 1000: 1: 1$, at $50{ }^{\circ} \mathrm{C}$ in $40 \mathrm{~mL}$

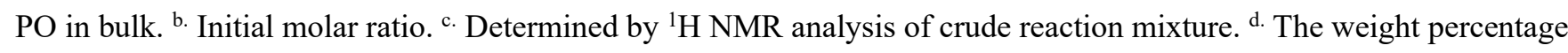
of by-product $\mathrm{cPC}$, determined by ${ }^{1} \mathrm{H}$ NMR analysis. ${ }^{\text {e. Turnover frequency, calculated by }(\mathrm{mol}} \mathrm{PO}$ to polyol $) /(\mathrm{mol} \mathrm{Al} \times \mathrm{h})$.

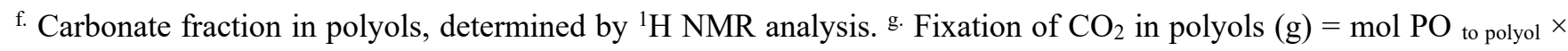
Carbonate $\% \times 44$. h. Determined by gel permeation chromatography in $\mathrm{CH}_{2} \mathrm{Cl}_{2}$ at $35{ }^{\circ} \mathrm{C}$, calibrated with polyethylene glycol standards. ${ }^{\text {i. }}$ Molar ratio of $[\mathrm{PO}] /[\mathrm{SA}]: 40 .{ }^{\mathrm{j} .}$ Molar ratio of $[\mathrm{PO}] /[\mathrm{SA}]:$ 32. ${ }^{\mathrm{k} .}$ Molar ratio of [PO]/[SA]: 25.6. ${ }^{1 .}$ Polymerization was run at $50{ }^{\circ} \mathrm{C}$ with molar ratio of [PO]:[SA]:[Al]:[PPNCl] $=25000: 500: 1: 1$, in a mixture of $20 \mathrm{~mL}$ $\mathrm{PO}$ and $20 \mathrm{~mL} \mathrm{CH}_{2} \mathrm{Cl}_{2}$. 

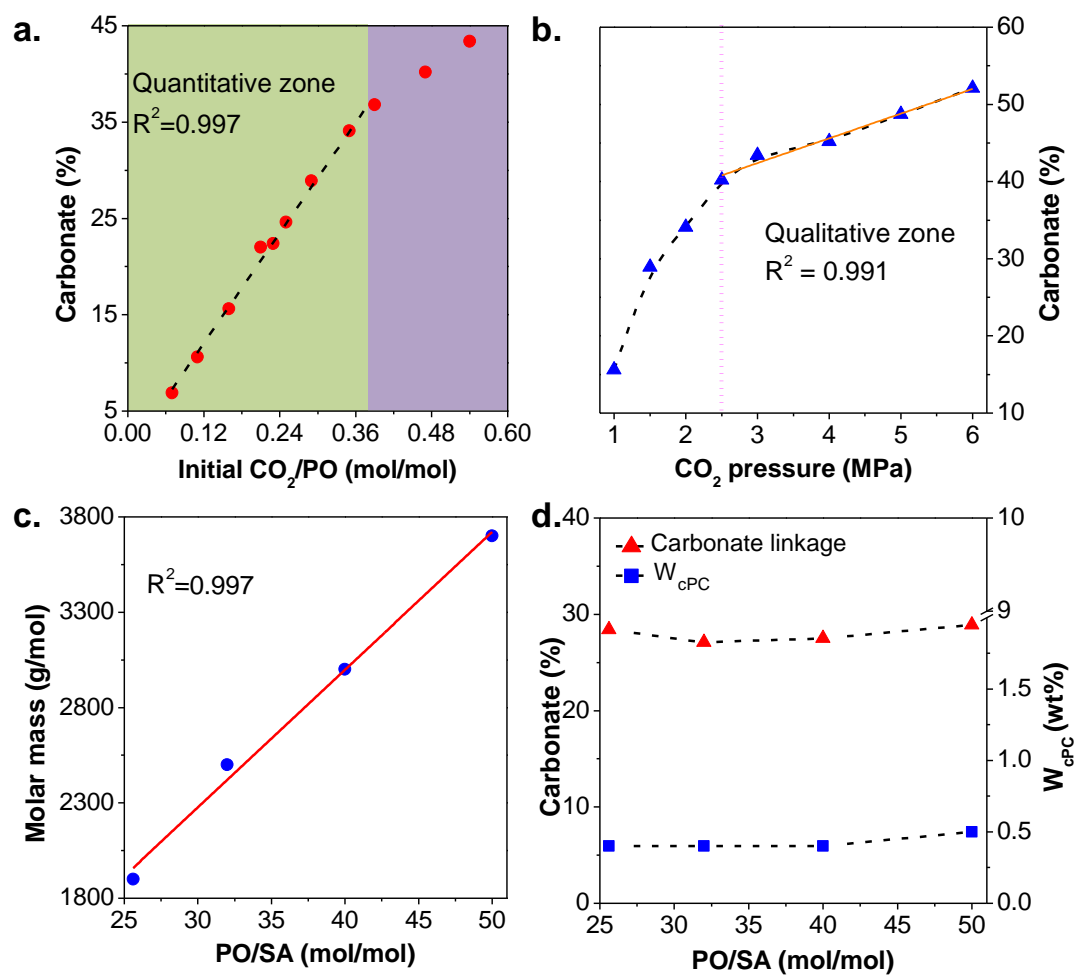

Figure 4. Precisely tuning the structure of $\mathrm{CO}_{2}$-polyol in the presence of CAT 1. a. Quantitative relationship between polyol composition and the feed ratio of $\mathrm{CO}_{2} / \mathrm{PO}$. b. Qualitative relationship between polyol composition and $\mathrm{CO}_{2}$ pressure, data were collected from Entry 3, 7, 11, 13, 15 of Table 1 and Entry 1, 2, 3 of Table S6. c. Control of molar mass by the feed ratio of PO/SA. d. Invariability of selectivity and composition under different ratio of PO/SA. The data of $\mathbf{c}$ and $\mathbf{d}$ were collected from Entry 7-10 of Table 1.

The relationship between apparent $\mathrm{CO}_{2}$ pressure and polyol composition was also studied. At $50{ }^{\circ} \mathrm{C}, 2 \mathrm{MPa} \mathrm{CO}_{2}$ pressure relatively corresponds to $\mathrm{CO}_{2} / \mathrm{PO}$ molar ratio of 0.35 , demarcating the "quantitative zone" (Fig. 4a). The pressure in Entry 13 and 15 of $C a$. $2.5 \mathrm{MPa}$ and $3 \mathrm{MPa}$ resulted in lower $\mathrm{CO}_{2}$ conversion (85.8\%, 79.1\%), while adding solvent can lower the viscosity of the medium and raise the solubility of $\mathrm{CO}_{2}$, facilitating increased $\mathrm{CO}_{2}$ conversion (Entry 14, Table 1). In addition, higher pressure i.e. excess $\mathrm{CO}_{2}$ reactions were also investigated (Table $\mathrm{S}_{\text {6) }} \mathrm{CO}_{2}$ pressure at $5 \mathrm{MPa}$ raised the carbonate linkage to $48.7 \%$, while a further increase to $6 \mathrm{MPa}$ reached the maximum at $52.1 \%$ yet with significant activity loss due to the gas expansion. Interestingly, there is a linear relationship between initial $\mathrm{CO}_{2}$ pressure and carbonate ratio from 2.5 $\mathrm{MPa}$ to $6 \mathrm{MPa}$, which forms the "qualitative zone". In general, in addition to quantitative prediction, the composition can also be qualitatively predicted by initial $\mathrm{CO}_{2}$ pressure (Fig. $4 \mathrm{~b}$ ).

Molar mass is another important parameter of $\mathrm{CO}_{2}$-polyols. Gel permeation chromatography (GPC) analyzed molar mass and distribution were calibrated with polyethylene glycol (PEG) as standards, generally used in characterization of low-molar-mass polyols. ${ }^{35}$ As shown in Entry 7-10, Table 1, by altering the amount of SA with fixed $\mathrm{CO}_{2} / \mathrm{PO}$ ratio, a series of $\mathrm{CO}_{2}$-polyols with identical composition, molar mass in the range of 1900-3700 g/mol were delicately prepared accompanied by invariant $\mathrm{cPC}$ content (Fig. 4d). The intact catalytic performance illustrated the advanced stability of CAT 1 while some organometallic catalysts have been reported to undergo deactivation in the presence of excess protic compounds. ${ }^{36}$ GPC results showed monomodal and narrow distributions $(<1.12)$ and the molar mass was linearly dependent on the feed of PO/SA with correlation coefficient $\left(\mathrm{R}^{2}\right)$ above 0.99 , demonstrating the characteristic "immortal" polymerization (Fig. 4c). ${ }^{37}$ The as-prepared $\mathrm{CO}_{2}$-polyols were analyzed by MALDI-ToF mass spectrometry, which 
disclosed that all peaks corresponded to poly(ether carbonate) with SA core in the center and hydroxyl functions in terminal (Fig. S35-40). In short, $\mathrm{CO}_{2}$-polyols can be precisely customized here by adjusting the feed of two monomers and CTA, to meet the different practical requirements.

\section{Kinetics study and mechanistic consideration.}

Firstly, we monitored the whole kinetics profiles of $\mathrm{CO}_{2}$-polyol formation under CAT 1 using in situ infrared spectroscopy. As shown in Fig. 5a, the carbonyl absorption at $C a .1800 \mathrm{~cm}^{-1}$ for $\mathrm{cPC}$ was absent throughout the reaction. The absorption at $C a .2335 \mathrm{~cm}^{-1}$ for $\mathrm{CO}_{2}$ reached the plateau prior to the absorption at $C a .827 \mathrm{~cm}^{-1}$ for PO, which disclosed that the $\mathrm{CO}_{2}$-involved polymerization directly switched to PO ROP at the late stage. Such in situ switch polymerization assured the quantitative $\mathrm{CO}_{2}$ transformation into polyols, which is sharply differentiated from the occurrence of depolymerization as observed in the mononuclear catalyst. The $k_{\mathrm{obs}}$ for carbonate and ether formation determined by the slope of linear part were 0.14 [abs. $/ \mathrm{h}]$ and 0.23 [abs. $/ \mathrm{h}]$, the ratio of which was somehow correlated with the maximum carbonate ratio in the "quantitative zone" $(0.14 /(0.14+0.23)=0.38)$. For careful calculation, ex situ aliquot ${ }^{1} \mathrm{H}$ NMR analysis was used to determine monomer numbers per chain, $\mathrm{CO}_{2}$ net fixation and carbonate ratio at different time intervals in two separate reactions at $0.8 \mathrm{MPa} / 2.2 \mathrm{MPa}$ pressure (Fig. $5 \mathrm{~b}$ ). In the first $6 \mathrm{~h}$ of the two reactions, the rate of $\mathrm{CO}_{2}$ insertion exhibits the same value $\left(\mathrm{Ca} .0 .4 \mathrm{CO}_{2} \mathrm{~g} / \mathrm{h}\right)$ yet with different carbonate linkage, illustrating the moderate deceleration of PO ROP by the increased $\mathrm{CO}_{2}$ feed. The $0.8 \mathrm{MPa}$ reaction finished $\mathrm{CO}_{2}$ consumption within $10.5 \mathrm{~h}$ left with $38 \%$ unreacted $\mathrm{PO}$ and the full conversion of PO was achieved at $20 \mathrm{~h}$. The $2.2 \mathrm{MPa}$ reaction which represented the edge of "quantitative zone", converted $4.6 \mathrm{~g} \mathrm{CO}_{2}$ within $12 \mathrm{~h}$ and the rest $0.5 \mathrm{~g} \mathrm{CO}_{2}$ still required another $12 \mathrm{~h}$ due to the highly viscous medium. As shown in Fig. $5 \mathrm{c}$, the molar mass increased smoothly with time and displayed monomodal distribution at each time interval with narrow PDI values $(<1.14)$. Meanwhile, the results of GPC analysis fitted well with ${ }^{1} \mathrm{H}$ NMR spectroscopy, demonstrating a well-controlled process. The analysis of molar mass also proved that after $\mathrm{CO}_{2}$ consumption excess $\mathrm{PO}$ continued to insert in the existed chains generating homogeneous poly(ether carbonate) in the end rather than regenerate a sole polyether chain.

In a related research by Williams and coworkers using a mononuclear chromium catalyst, equimolar feed of cyclic anhydride and epoxide formed polyester, while excess epoxide afforded switchable polymerization between ROCOP and ROP producing block copolymers with versatile architectures. ${ }^{38}$ Unfortunately, when it came to $\mathrm{PO} / \mathrm{CO}_{2}$, the catalytic cycle was likely to shift to "back-biting" from propagation as illustrated in Fig. 3. In this work, the successful quantitative $\mathrm{CO}_{2}$ polymerization is undoubtedly attributed to CAT 1 which shares the same structure of active site with mono 1 but functions entirely different. To better understand the advanced multisite catalysis of CAT 1, we calculated the order in $\mathrm{CO}_{2}$, epoxide and catalyst for the formation of PPC by determining the initial rates (slopes of absorbance at $\mathrm{Ca} .1745$ $\mathrm{cm}^{-1}$ versus time at conversion below 15\%) in controlled series of experiments. Since SA with poor solubility in PO, is gradually dissolved by the enchainment of PO, while PEG dissolves well in PO whose absorption at Ca. $1104 \mathrm{~cm}^{-1}$ flattened very fast as monitored. Therefore, PEG was used as CTA and initial rates were measured without interference. A zero order in $\mathrm{CO}_{2}$ was observed over the range of 0.5-2.5 MPa pressure, revealing that the insertion of $\mathrm{CO}_{2}$ is not ratedetermining for carbonate formation (Fig. 6a). Surprisingly, the initial rates at 3.0-4.6 MPa were only about one-third of those at 0.5-2.5 $\mathrm{MPa}$ due to the gas expansion. As previously reported, $\mathrm{CO}_{2}$ insertion is always fast and zero-order dependence in most $\mathrm{CO}_{2}$ /epoxide ROCOP catalysis. ${ }^{16-18}$ While Rieger and coworkers found that under a di-zinc catalyst the $\mathrm{CO}_{2}$ insertion was first-order below $2.5 \mathrm{MPa}$ and shifted to zero-order at higher pressure. ${ }^{39}$ Similarly, we here found that 2.5 MPa was the boundary between the "quantitative zone" and the "qualitative zone", and yet the distinct shift for 
the reaction order in $\mathrm{CO}_{2}$ was not observed around 2.5 $\mathrm{MPa}$. In the "quantitative zone", the initial rates only fluctuated with $\mathrm{CO}_{2}$ pressure within the range of $\pm 13.5 \%$. Therefore, the critical value of the zone also reflected the intrinsic selectivity of CAT 1 between ROP and ROCOP as disclosed before. In the "qualitative zone", with the increase in $\mathrm{CO}_{2}$ pressure, the rate of ROP significantly dropped while ROCOP still exhibited the similar rate, which resulted in such coincidentally linear dependency of polyol composition on the $\mathrm{CO}_{2}$ pressure.
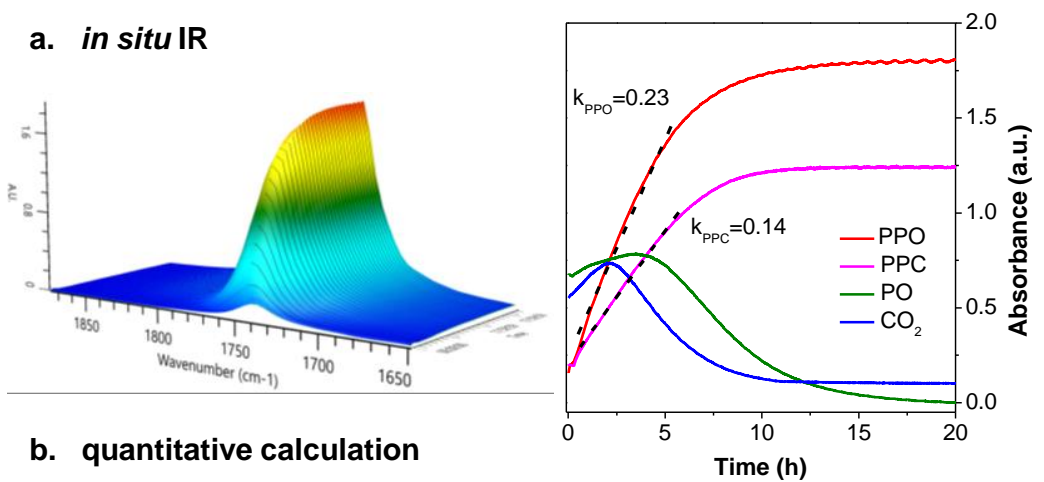

b. quantitative calculation
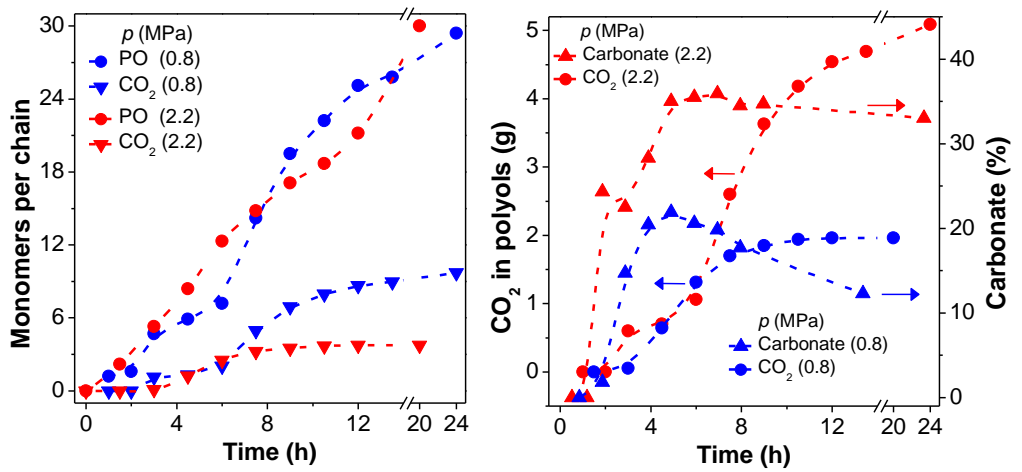

c. molar mass
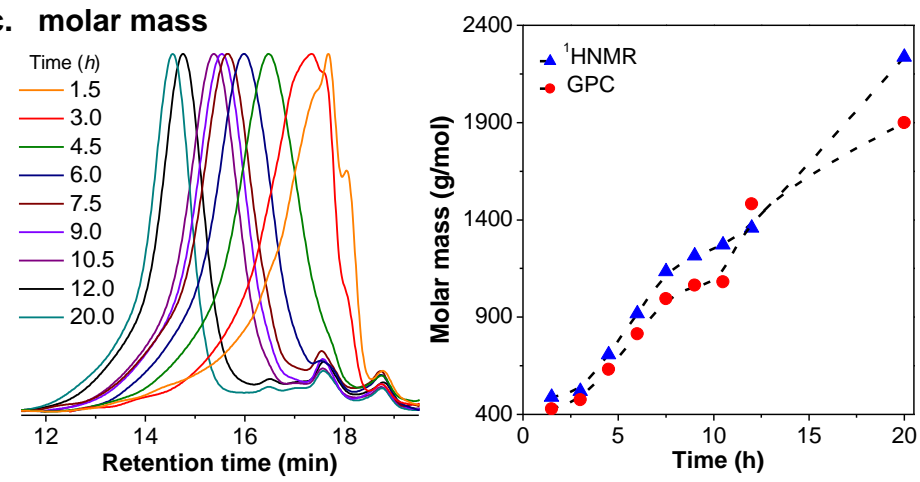

Figure 5. Monitoring CAT 1 catalyzed quantitative $\mathrm{CO}_{2}$ polymerization. a. Reaction profiles monitored by in situ IR and the resulting three-dimensional stack plot of IR spectra. Profiles of the absorbance at $C a .2335 \mathrm{~cm}^{-1}$ corresponds to $v(\mathrm{C}=\mathrm{O})$ from $\mathrm{CO}_{2}$. Profiles of the absorbance at $\mathrm{Ca} .827 \mathrm{~cm}^{-1}$ corresponds to $v(\mathrm{C}-\mathrm{O})$ from PO. Reaction condition: $\mathrm{PO} /[\mathrm{SA}] /[\mathrm{Al}] / \mathrm{PPNCl}=50000 / 1000 / 1 / 1$ (molar ratio), $50{ }^{\circ} \mathrm{C}, 1.5 \mathrm{MPa} \mathrm{CO}_{2}$ pressure, $30 \mathrm{~mL} \mathrm{PO}$ in bulk. The final crude is also determined by ${ }^{1} \mathrm{H}$ NMR analysis: conv. of $\mathrm{PO} \%>99 \%, \mathrm{~W}_{\mathrm{cPC}} \% 0.2 \%$, Carbonate $\% 27.1 \%$. b. Quantitative calculation by ${ }^{1} \mathrm{H}$ NMR determining sampled aliquots at different time intervals, presented by plots of numbers of monomer enchained, mass of $\mathrm{CO}_{2}$ in polyols, and carbonate linkage, versus time. Reactions were carried out in the autoclave equipped with a sampling valve, with $\mathrm{PO} /[\mathrm{Al}] / \mathrm{PPNCl}=50000 / 1 / 1 \& \mathrm{PO} / \mathrm{SA}=30$ (molar ratio), $50{ }^{\circ} \mathrm{C}, 0.8 / 2.2$ $\mathrm{MPa} \mathrm{CO}_{2}$ pressure, $25 \mathrm{~mL}$ PO in bulk. c. Plots of $M_{\mathrm{n}}$ versus time and GPC curves of sampled aliquots from $0.8 \mathrm{MPa}$ reaction in $\mathbf{b}$. 
The double logarithmic plot of initial rates and PO concentration gave the reaction order in PO as the slope of the plot. As shown in Fig. 6b, a first-dependence for PO was clearly demonstrated in the concentration of 1.4-10.5 M in dichloromethane. This strongly suggests the ring-opening of PO attacked by the carbonate intermediate is the ratedetermining step rather than $\mathrm{CO}_{2}$ insertion into the alkoxide. Next, the plot of $\ln \left(k_{\mathrm{obs}}\right)$ versus $\ln (\mathrm{cat})$ gave a slope of 0.9 , indicating a first-order dependence in CAT 1 within experimental error (Fig. 6c). ${ }^{40}$ This stands in contrast to the order between 1 and 2 as observed in most mononuclear catalysts, ${ }^{16}$ indicating the occurrence of intramolecular metal-metal cooperativity within one oligomeric catalyst chain as previously evidenced in the multinuclear $\mathrm{Co}$ (III) salen catalyzed hydrolytic kinetic resolution of epoxides ${ }^{41,42}$ and a recent multimetallic $\mathrm{CO}_{2}$ /epoxide ROCOP catalysis ${ }^{43}$. To determine the activation energy $\left(E_{\mathrm{a}}\right)$, initial rates were measured in CAT 1 involved controlled experiments at $50{ }^{\circ} \mathrm{C}, 60{ }^{\circ} \mathrm{C}, 70^{\circ} \mathrm{C}$, $80{ }^{\circ} \mathrm{C}$, respectively. Fig. $6 \mathrm{~d}$ depicted the Arrhenius plots for the formation of PPC while the absorption at $C a .1800 \mathrm{~cm}^{-1}$ for cPC was not observable in such temperature range. The $E_{\mathrm{a}}$ for PPC was $34.2 \mathrm{~kJ} / \mathrm{mol}$ in the presence of CAT 1, while mono 1 showed significant higher $E_{\mathrm{a}}$ value of $44.7 \mathrm{~kJ} / \mathrm{mol}$ for PPC (Fig. S51). The $\Delta E_{\mathrm{a}}$ of $10.5 \mathrm{~kJ} / \mathrm{mol}$ correlates well with the differentiated catalytic performance as observed before.
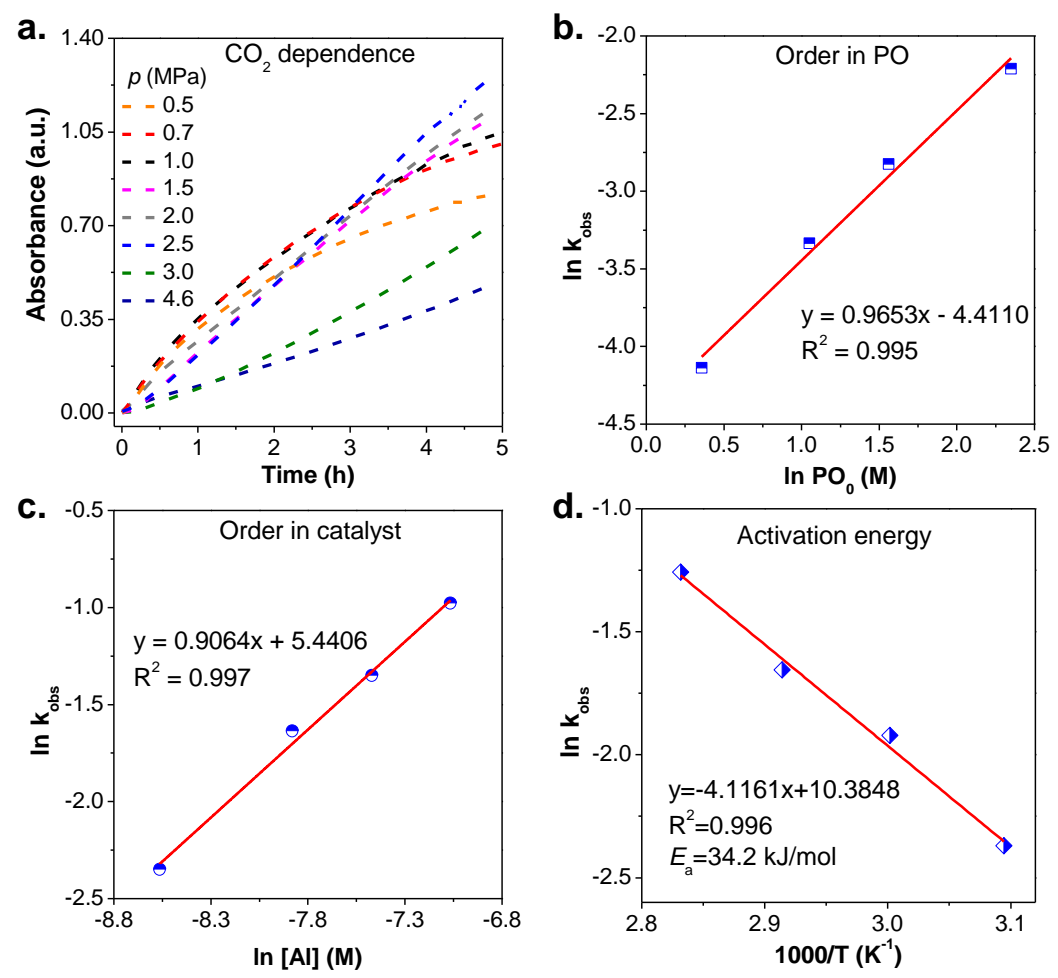

Figure 6. Kinetics studies of CAT 1 for the formation of PPC determined by in situ IR. a. The dependence of initial rates on $\mathrm{CO}_{2}$ pressure. b. Order in PO. c. Order in catalyst. d. Arrhenius plot.

In addition to Arrhenius method, the Eyring analysis was also involved to determine the activation parameters for further insight into the catalysis (Fig. S53). ${ }^{44,45}$ With CAT 1 (Fig. S49), the enthalpy of activation ( $\Delta \mathrm{H}^{\ddagger}$ ) values for PPC and PPO were determined to be $31.4,42.0 \mathrm{~kJ} / \mathrm{mol}$ and the entropy of activation $\left(\Delta \mathrm{S}^{\ddagger}\right)$ values for PPC and PPO were $167.9,-144.3 \mathrm{~J} /\left(\mathrm{mol}^{*} \mathrm{~K}\right)$, respectively. Overall, the transition state Gibbs free energy $\left(\Delta \mathrm{G}^{\ddagger}\right)$ values were $89.0 \mathrm{~kJ} / \mathrm{mol}$ for PPC and $91.5 \mathrm{~kJ} / \mathrm{mol}$ for PPO (at $70{ }^{\circ} \mathrm{C}$ ). This similarity in $\Delta \mathrm{G}^{*}$ values support the fact that CAT 1 exhibits comparative rates of ROP and ROCOP from a thermodynamic perspective. Classic metal complexes like porphyrin and salen catalysts 
normally produce polycarbonates with trivial ether linkage, while in striking difference, oligomeric CAT 1 can produce ether-rich $\mathrm{CO}_{2}$-polyols which extends the employment of metal-based $\mathrm{CO}_{2}$ ROCOP catalysts previously only for biodegradable plastics to the manufacturing of polyurethanes. For comparison, the Eyring analysis was also applied for the case of mono 1 in similar controlled temperature-dependent experiments achieving the following activation parameters for PPC: $\Delta \mathrm{H}^{\star}$ of $41.9 \mathrm{~kJ} / \mathrm{mol}, \Delta \mathrm{S}^{\ddagger}$ of $-158.4 \mathrm{~J} /\left(\mathrm{mol} * \mathrm{~K}\right.$ ) and $\Delta \mathrm{G}^{\ddagger}$ (at $70{ }^{\circ} \mathrm{C}$ ) of $96.2 \mathrm{~kJ} / \mathrm{mol}$ (Fig. S52). Since $\mathrm{CO}_{2}$ is zero-order dependence in PPC formation, these activation parameters are supposed to be consistent with the transition state involving a carbonate end attacking the activated PO (Fig. S54). With mono 1 the replacement of carbonate by $\mathrm{PO}$ in the coordinating site and the ring-opening of $\mathrm{PO}$ by carbonate proceed within one metal, while CAT 1 allows that both anionic carbonate and PO are well stabilized in an intra- bimetallic manner and such Al-Al synergy is just a random permutation along the oligomeric chain. Such difference is substantiated by the $\Delta \mathrm{H}^{\ddagger}$ value which is largely related to the stability of such transition state. The reduced enthalpy barrier with CAT 1 accelerates the PO ring-opening step which leads to the high activity of the whole catalysis. On the other hand, the oligomer catalyst CAT 1 accumulates monomers and propagating chains in a confined space, which decreases the degrees of freedom resulting in more entropic loss. Nevertheless, in the range of common operating temperature $\left(25-100{ }^{\circ} \mathrm{C}\right)$, the use of $\mathbf{C A T} \mathbf{1}$ is always thermodynamically favored and $\Delta\left(\Delta \mathrm{G}^{\ddagger}\right)$ of CAT 1 and mono 1 is $6.9-7.6 \mathrm{~kJ} / \mathrm{mol}$ due to the remarkable enthalpic benefit.

In general, quantitative $\mathrm{CO}_{2}$ polymerization faces overlapped difficulties from low $\mathrm{CO}_{2}$ pressure, the requirement of full conversion, and the use of protic compounds. The above discussion explains how CAT 1 facilitates the transformation of quantitative $\mathrm{CO}_{2}$ into polyols. Last but not least, another question is how to preserve such transformation afterwards, i.e., why CAT 1 realizes the switch catalysis from ROCOP to ROP while mono 1 shifted to back-biting reaction under the same condition. To this end, the activation parameters with mono 1 at the late stage were also studied. As shown in Fig. 7, when $\mathrm{CO}_{2}$ is insufficient at the late stage, the catalytic resting state stalls at metalalkoxide $(R S)$, where three pathways may occur subsequently in the presence of excess PO as the monomer and solvent at the same time. In Path A, RS undergoes 1) the dissociation of polyol from the metal and 2) intramolecular "back-biting" between alkoxide end and adjacent carbonyl to give TS A. At last, cPC is afforded after releasing the remained chain which is likely to be coordinated back to the metal center or to diffuse into the bulk solution. With mono 1, the values of $\Delta \mathrm{H}^{\ddagger}$ and $\Delta \mathrm{G}^{\star}\left(\right.$ at $\left.70{ }^{\circ} \mathrm{C}\right)$ were measured to be $40.5,87.7 \mathrm{~kJ} / \mathrm{mol}$ for such depolymerization. These activation parameters are significantly lower compared to those for initial $\mathrm{cPC}$ formation (the values of $\Delta \mathrm{H}^{\ddagger}$ and $\Delta \mathrm{G}^{*}$ at $70{ }^{\circ} \mathrm{C}$ were $57.9,96.6$ $\mathrm{kJ} / \mathrm{mol}$ ) under the same catalyst. The values are in line with the result where $\mathrm{cPC}$ is formed much faster by depolymerization from PPC at the late stage than coupling reaction in the beginning. In Path B, the alkoxide is first substituted by a new PO from the coordinating site and then directly attack it to realize enchainment. The activation parameters to reach TS $B$ with mono 1 were much higher: $\Delta \mathrm{H}^{\star}$ of $55.0 \mathrm{~kJ} / \mathrm{mol}, \Delta \mathrm{G}^{\ddagger}$ (at $70{ }^{\circ} \mathrm{C}$ ) of $100.7 \mathrm{~kJ} / \mathrm{mol}$. As evidenced in the kinetic studies with mono 1, the PO enchainment via Path B is dominated by the depolymerization via Path A. In sharp contrast, in Path C, CAT 1 reached $T S C$ with $\Delta \mathrm{H}^{\ddagger}$ of $42.0 \mathrm{~kJ} / \mathrm{mol}, \Delta \mathrm{G}^{\ddagger}\left(\right.$ at $70{ }^{\circ} \mathrm{C}$ ) of $91.5 \mathrm{~kJ} / \mathrm{mol}$ as mentioned before, which was much lower than those of monometallic Path B. The reduced enthalpy is still the main contributor, since CAT 1 with multiple Al centers assures the involvement of two metals (one for PO activation and another for the stabilization of the alkoxide end) in the attack of PO which effectively stabilizes the anionic transition state TS C. A previous literature even reported that the shorter distanced bimetallic pathway had more prominent promotion in PO ROP than PO/CO 2 ROCOP. ${ }^{46}$ Therefore, CAT 1 successfully shifts to ROP process from $R S$ via Path $\mathrm{C}$ and thus sequesters the before-polymerized $\mathrm{CO}_{2}$ once for all. Besides, from a macroscopic perspective, a feature of catalytic chain-transfer polymerization is that one metal serves as the active site for multiple propagation events ${ }^{47}$ which 
greatly amplifies the diversity since free-base chains are more unstable and prone to depolymerize as previously illustrated by Darensbourg. ${ }^{48}$ Hence, the isolated active sites also cause such uncontrolled catalysis in the presence of common mononuclear catalyst like mono 1. In terms of CAT 1, the loading is more extreme which results in one metal versus $C a$. 1,000 polyols (Table 1). Nevertheless, the accumulation effect of CAT 1 creates more possibilities for bimetallic interactions, and thus the swap between dormant chains and activated chains is tremendously accelerated which also stabilizes the dormant chains from "back-biting".

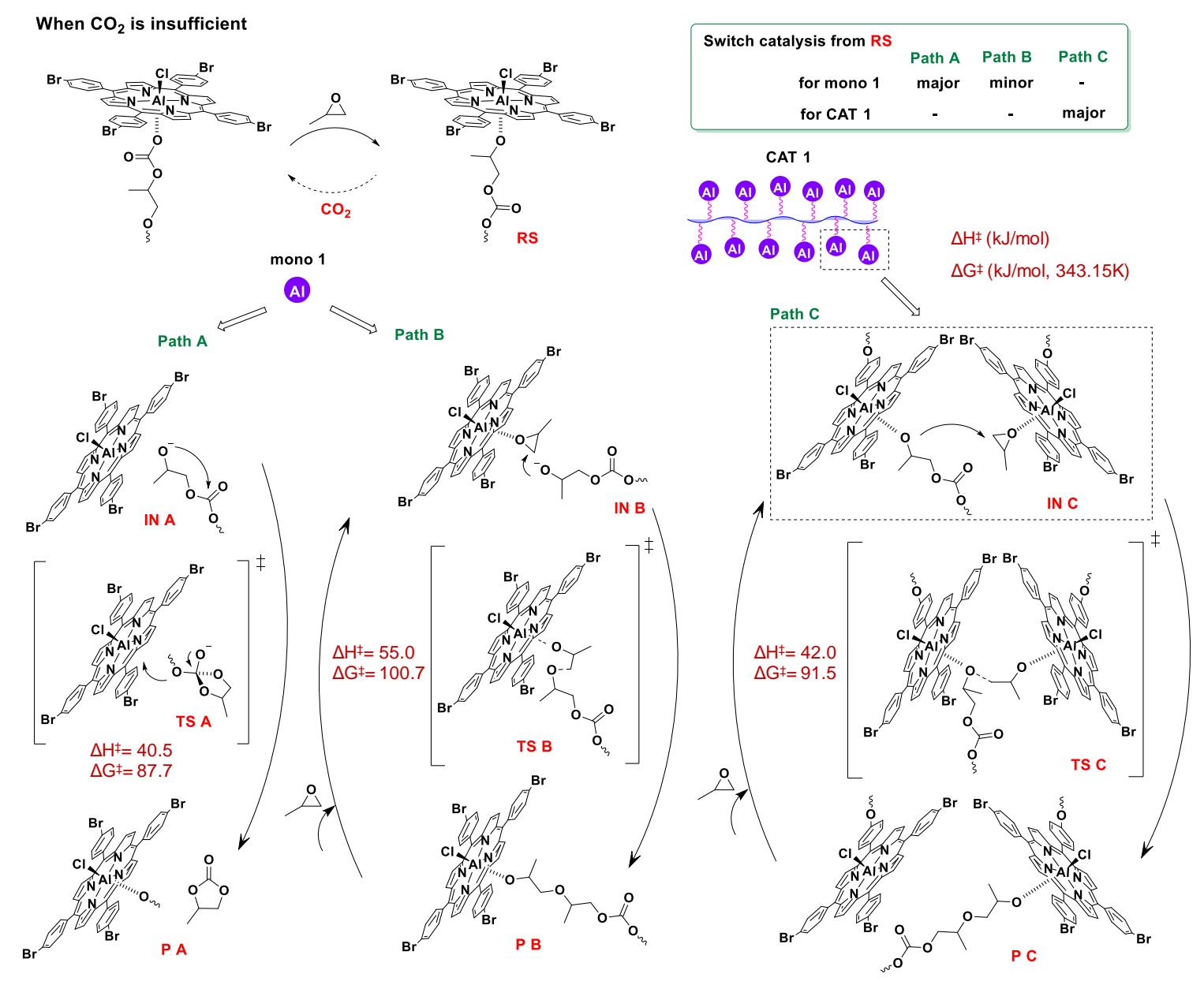

Figure 7. Proposed pathways from Al-alkoxide resting state at high $\mathrm{CO}_{2}$ conversion.

\section{Conclusion}

Different from high pressure condition normally required in $\mathrm{CO}_{2}$ transformation, and also different from the pursuit of absolute ambient pressure reaction, we establish an on-demand $\mathrm{CO}_{2}$ fixation, which is the first time for quantitative $\mathrm{CO}_{2}$ polymerization, tuning the composition of polyols by accurate $\mathrm{CO}_{2}$ feed. Kinetic study reveals that under substoichiometric $\mathrm{CO}_{2}$, CAT 1 allows the in situ switch catalysis between $\mathrm{CO}_{2}$-involved copolymerization and PO ROP when $\mathrm{CO}_{2}$ was fully reacted, while common mononuclear catalysts shift the selectivity at high $\mathrm{CO}_{2}$ conversion producing a mixture of polyol and cyclic carbonate. The success is attributed to the intra- multimetallic cooperativity of CAT 1, which increases the rate of monomer enchainment, stabilizes the key intermediates and blocks the "back-biting" pathway. The findings presented here provide mutual benefit for quantitative $\mathrm{CO}_{2}$ transformation and precise polymer synthesis, which also elicits a question how precise catalysis can offer better opportunities for the development of green chemistry. 


\section{Data availability}

Detailed experimental procedures, the characterization of all the synthesized compounds and polymers, other data mentioned in the manuscript are provided in the Supporting Information.

\section{Author information}

Corresponding Author

*E-mail: xhwang@ciac.ac.cn (X.W.).

ORCID identification number(s)

Xianhong Wang: 0000-0002-4228-705X

\section{Conflict of interest}

The authors declare no competing financial interest.

\section{Acknowledgement}

The authors greatly appreciated the financial support from Fundamental Science Center projector in National Natural Science Foundation of China (Grant No. 51988102) and Key Research Program of Frontier Sciences, CAS (Grant No .QYZDJ-SSW-JSC017).

\section{References:}

1 Hepburn, C. et al. The technological and economic prospects for $\mathrm{CO}_{2}$ utilization and removal. Nature 575, 8797 (2019).

2 Artz, J. et al. Sustainable Conversion of Carbon Dioxide: An Integrated Review of Catalysis and Life Cycle Assessment. Chem. Rev. 118, 434-504 (2018).

3 Mahoutian, M. \& Shao, Y. Production of cement-free construction blocks from industry wastes. J. Clean. Prod. 137, 1339-1346 (2016).

4 Song, Q. W., Zhou, Z. H., Yin, H. \& He, L. N. Silver(I)-Catalyzed Synthesis of $\beta$-Oxopropylcarbamates from Propargylic Alcohols and $\mathrm{CO}_{2}$ Surrogate: A Gas-Free Process. ChemSusChem 8, 3967-3972 (2015).

5 De Luna, P. et al. Catalyst electro-redeposition controls morphology and oxidation state for selective carbon dioxide reduction. Nat. Catal. 1, 103-110 (2018).

6 Zheng, $\mathrm{X}$. et al. Theory-guided $\mathrm{Sn} / \mathrm{Cu}$ alloying for efficient $\mathrm{CO}_{2}$ electroreduction at low overpotentials. Nat. Catal. 2, 55-61 (2019).

7 Klaus, S., Lehenmeier, M. W., Anderson, C. E. \& Rieger, B. Recent advances in $\mathrm{CO}_{2} /$ epoxide copolymerization-New strategies and cooperative mechanisms. Coord. Chem. Rev. 255, 1460-1479 (2011).

8 Nakano, R., Ito, S. \& Nozaki, K. Copolymerization of carbon dioxide and butadiene via a lactone intermediate. Nat. Chem. 6, 325-331 (2014).

9 Huang, R. et al. Deciphering key intermediates in the transformation of carbon dioxide into heterocyclic products. Nat. Catal. 2, 62-70 (2019).

10 Qin, Y., Guo, H., Sheng, X., Wang, X. \& Wang, F. An aluminum porphyrin complex with high activity and selectivity for cyclic carbonate synthesis. Green Chem. 17, 2853-2858 (2015).

11 Sujith, S., Min, J. K., Seong, J. E., Na, S. J. \& Lee, B. Y. A Highly Active and Recyclable Catalytic System for 
$\mathrm{CO}_{2}$ /Propylene Oxide Copolymerization. Angew. Chem. Int. Ed. 47, 7306-7309 (2008). Anastas, P. \& Eghbali, N. Green Chemistry: Principles and Practice. Chem. Soc. Rev. 39, 301-312 (2010). Burkart, M. D., Hazari, N., Tway, C. L. \& Zeitler, E. L. Opportunities and Challenges for Catalysis in Carbon Dioxide Utilization. ACS Catal. 9, 7937-7956 (2019).

Langanke, J. et al. Carbon dioxide $\left(\mathrm{CO}_{2}\right)$ as sustainable feedstock for polyurethane production. Green Chem. 16, 1865-1870 (2014).

Deacy, A. C., Moreby, E., Phanopoulos, A. \& Williams, C. K. Co(III)/Alkali-Metal(I) Heterodinuclear Catalysts for the Ring-Opening Copolymerization of $\mathrm{CO}_{2}$ and Propylene Oxide. J. Am. Chem. Soc. 142, 19150-19160 (2020).

6 Moore, D. R., Cheng, M., Lobkovsky, E. B. \& Coates, G. W. Mechanism of the Alternating Copolymerization of Epoxides and $\mathrm{CO}_{2}$ Using $\beta$-Diiminate Zinc Catalysts: Evidence for a Bimetallic Epoxide Enchainment. $J$. Am. Chem. Soc. 125, 11911-11924 (2003).

Jutz, F., Buchard, A., Kember, M. R., Fredriksen, S. B. \& Williams, C. K. Mechanistic Investigation and Reaction Kinetics of the Low-Pressure Copolymerization of Cyclohexene Oxide and Carbon Dioxide Catalyzed by a Dizinc Complex. J. Am. Chem. Soc. 133, 17395-17405 (2011).

8 Yang, G.-W., Zhang, Y.-Y., Xie, R. \& Wu, G. P. Scalable Bifunctional Organoboron Catalysts for Copolymerization of $\mathrm{CO}_{2}$ and Epoxides with Unprecedented Efficiency. J. Am. Chem. Soc. 142, 12245-12255 (2020).

19 Qin, Z. Q., Thomas, C. M., Lee, S. \& Coates, G. W. Cobalt-based complexes for the copolymerization of propylene oxide and $\mathrm{CO}_{2}$ : Active and selective catalysts for polycarbonate synthesis. Angew. Chem. Int. Ed. 42, 5484-5487 (2003).

0 Lu, X.-B. et al. Design of Highly Active Binary Catalyst Systems for $\mathrm{CO}_{2} /$ Epoxide Copolymerization: Polymer Selectivity, Enantioselectivity, and Stereochemistry Control. J. Am. Chem. Soc. 128, 1664-1674 (2006).

Nakano, K., Kamada, T. \& Nozaki, K. Selective Formation of Polycarbonate over Cyclic Carbonate: Copolymerization of Epoxides with Carbon Dioxide Catalyzed by a Cobalt(III) Complex with a Piperidinium End-Capping Arm. Angew. Chem. Int. Ed.45, 7274-7277 (2006).

2 Robertson, N. J. et al. Two-dimensional double metal cyanide complexes: highly active catalysts for the homopolymerization of propylene oxide and copolymerization of propylene oxide and carbon dioxide. Dalton Trans. 5390-5395 (2006).

Liu, S. J., Qin, Y. S., Chen, X. S., Wang, X. H. \& Wang, F. S. One-pot controllable synthesis of oligo(carbonateether) triol using a Zn-Co-DMC catalyst: the special role of trimesic acid as an initiation-transfer agent. Polym. Chem. 5, 6171-6179 (2014).

4 Subhani, M. A., Kohler, B., Gurtler, C., Leitner, W. \& Müller, T. E. Transparent Films from $\mathrm{CO}_{2}$-Based Polyunsaturated Poly(ether carbonate)s: A Novel Synthesis Strategy and Fast Curing. Angew. Chem. Int. Ed. 55, 5591-5596 (2016).

5 Deacy, A. C., Kilpatrick, A. F. R., Regoutz, A. \& Williams, C. K. Understanding metal synergy in heterodinuclear catalysts for the copolymerization of $\mathrm{CO}_{2}$ and epoxides. Nat. Chem. 12, 372-380 (2020).

6 Nagae, H. et al. Lanthanide Complexes Supported by a Trizinc Crown Ether as Catalysts for Alternating Copolymerization of Epoxide and $\mathrm{CO}_{2}$ : Telomerization Controlled by Carboxylate Anions. Angew. Chem. Int. Ed. 57, 2492-2496 (2018). 

Intramolecular Cooperativity for the Synthesis of $\mathrm{CO}_{2}$-Based Polymers. ACS Catal. 9, 8669-8676 (2019). Sugimoto, H., Aida, T. \& Inoue, S. Ring-opening polymerizations of lactone and epoxide initiated with aluminum complexes of substituted tetraphenylporphyrins. Molecular design of highly active initiators. Macromolecules 23, 2869-2875 (1990).

29 Deng, J. et al. Aluminum porphyrins with quaternary ammonium halides as catalysts for copolymerization of cyclohexene oxide and $\mathrm{CO}_{2}$ : metal-ligand cooperative catalysis. Chem. Sci. 11, 5669-5675 (2020). Subhani, M. A., Gürtler, C., Leitner, W. \& Müller, T. E. Nanoparticulate $\mathrm{TiO}_{2}$-Supported Double Metal Cyanide Catalyst for the Copolymerization of $\mathrm{CO}_{2}$ with Propylene Oxide. Eur. J. Inorg. Chem. 2016, 1944-1949 (2016).

31 Gao, Y. G., Gu, L., Qin, Y. S., Wang, X. H. \& Wang, F. S. Dicarboxylic acid promoted immortal copolymerization for controllable synthesis of low-molecular weight oligo(carbonate-ether) diols with tunable carbonate unit content. J. Polym. Sci., Part A: Polym. Chem. 50, 5177-5184 (2012).

Meys, R., Katelhon, A. \& Bardow, A. Towards sustainable elastomers from $\mathrm{CO}_{2}$ : life cycle assessment of carbon capture and utilization for rubbers. Green Chem. 21, 3334-3342 (2019).

33 Pohl, M. et al. Dynamics of Polyether Polyols and Polyether Carbonate Polyols. Macromolecules 49, 8995-9003 (2016).

34 Gong, R. et al. Terminal Hydrophilicity-Induced Dispersion of Cationic Waterborne Polyurethane from $\mathrm{CO}_{2}-$ Based Polyol. Macromolecules 53, 6322-6330 (2020).

35 Schömer, M., Seiwert, J. \& Frey, H. Hyperbranched Poly(propylene oxide): A Multifunctional BackboneThermoresponsive Polyether Polyol Copolymer. ACS Macro Lett. 1, 888-891 (2012).

$36 \mathrm{Na}, \mathrm{S}$. J. et al. Elucidation of the Structure of a Highly Active Catalytic System for $\mathrm{CO}_{2} /$ Epoxide Copolymerization: A salen-Cobaltate Complex of an Unusual Binding Mode. Inorg. Chem. 48, 10455-10465 (2009).

37 Asano, S., Aida, T. \& Inoue, S. 'Immortal' polymerization. Polymerization of epoxide catalysed by an aluminium porphyrin-alcohol system. J. Chem. Soc., Chem Commun. 1148-1149 (1985).

38 Stößer, T., Sulley, G. S., Gregory, G. L. \& Williams, C. K. Easy access to oxygenated block polymers via switchable catalysis. Nat. Commun. 10, 2668 (2019).

39 Lehenmeier, M. W. et al. Flexibly tethered dinuclear zinc complexes: a solution to the entropy problem in $\mathrm{CO}_{2}$ /epoxide copolymerization catalysis? Angew. Chem. Int. Ed. 52, 9821-9826 (2013).

40 Thevenon, A. et al. Indium Catalysts for Low-Pressure $\mathrm{CO}_{2} /$ Epoxide Ring-Opening Copolymerization: Evidence for a Mononuclear Mechanism? J. Am. Chem. Soc. 140, 6893-6903 (2018).

41 Ready, J. M. \& Jacobsen, E. N. Highly Active Oligomeric (salen)Co Catalysts for Asymmetric Epoxide RingOpening Reactions. J. Am. Chem. Soc. 123, 2687-2688 (2001).

42 Zhu, X., Venkatasubbaiah, K., Weck, M. \& Jones, C. W. Kinetic Evaluation of Cooperative [Co(salen)] Catalysts in the Hydrolytic Kinetic Resolution of rac-Epichlorohydrin. Chem CatChem 2, 1252-1259 (2010).

43 Asaba, H. et al. Alternating Copolymerization of $\mathrm{CO}_{2}$ and Cyclohexene Oxide Catalyzed by Cobalt-Lanthanide Mixed Multinuclear Complexes. Inorg. Chem. 59, 7928-7933 (2020).

44 Switzer, J. M. et al. Quantitative Modeling of the Temperature Dependence of the Kinetic Parameters for Zirconium Amine Bis(Phenolate) Catalysts for 1-Hexene Polymerization. ACS Catal. 8, 10407-10418 (2018).

45 Darensbourg, D. J., Moncada, A. I., Choi, W. \& Reibenspies, J. H. Mechanistic Studies of the Copolymerization 
Reaction of Oxetane and Carbon Dioxide to Provide Aliphatic Polycarbonates Catalyzed by (Salen)CrX Complexes. J. Am. Chem. Soc. 130, 6523-6533 (2008).

46 Ohkawara, T., Suzuki, K., Nakano, K., Mori, S. \& Nozaki, K. Facile estimation of catalytic activity and selectivities in copolymerization of propylene oxide with carbon dioxide mediated by metal complexes with planar tetradentate ligand. J. Am. Chem. Soc. 136, 10728-10735 (2014).

47 Li, Y. et al. Carbon dioxide-based copolymers with various architectures. Prog. Polym. Sci. 82, 120-157 (2018).

48 Darensbourg, D. J. Comments on the depolymerization of polycarbonates derived from epoxides and carbon dioxide: A mini review. Polym. Degrad. Stab. 149, 45-51 (2018). 
Table of Contents

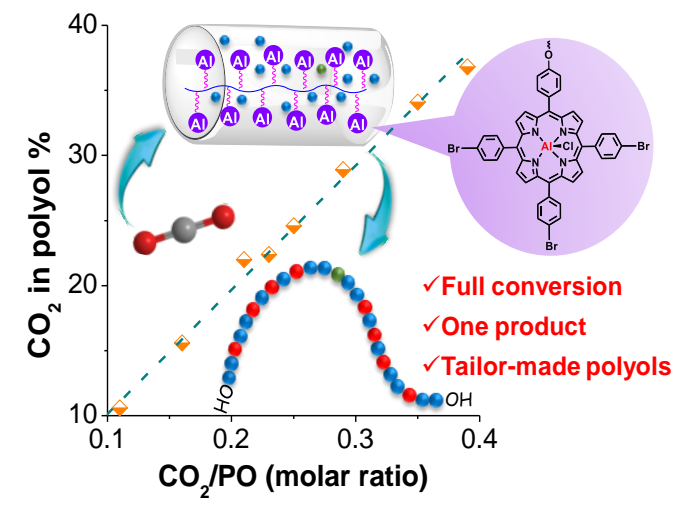

